Shuichi Iwashita $\cdot$ Kumiko Koyama

Yusuke Nakamura

\title{
VNTR sequence on human chromosome 11p15 that affects transcriptional activity
}

\begin{abstract}
We found a variable number of tandem repeat (VNTR) sequence with 38-bp repetitive units in the promoter region of a gene of unknown function on human chromosome $11 \mathrm{p} 15$. Polymerase chain reaction amplification of this VNTR sequence using genomic DNA from 80 unrelated individuals revealed two common alleles, one with 10 (79\% allelic frequency) and the other with 14 (14\% allelic frequency) repetitive units, and two rare alleles with $22(3 \%)$ or $30(3 \%)$ repetitive units. We investigated whether differences in the length of this VNTR sequence would affect transcriptional activity of a heterologous promoter by transient transfection to NEC8, embryonal carcinoma cells derived from testis. The activity of the promoter was suppressed significantly when the VNTR region was cloned upstream, in a manner dependent on the number of repeats present in the VNTR sequence. The results implied that this polymorphic VNTR sequence might function as transcriptional regulator in $11 \mathrm{p} 15$, with differences in the number of repetitive units influencing efficiency of transcription of the gene lying downstream.
\end{abstract}

Key words VNTR $\cdot 11 \mathrm{p} 15 \cdot$ Transcriptional regulator · Polymorphism $\cdot$ Testis

\section{Introduction}

VNTR (variable number of tandem repeat) markers, also called single-copy minisatellites, were originally isolated from human DNA as highly informative restriction frag-

S. Iwashita $\cdot$ K. Koyama $\cdot$ Y. Nakamura $(\triangle)$

Laboratory of Molecular Medicine, Human Genome Center,

Institute of Medical Science, The University of Tokyo, 4-6-1

Shirokanedai, Minato-ku, Tokyo 108-8639, Japan

Tel. +81-3-5449-5372; Fax +81-3-5449-5433

e-mail: yusuke@ims.u-tokyo.ac.jp ment length polymorphisms that were useful for linkage analyses because of their high degree of polymorphism among individuals (Nakamura et al. 1987). Evidence has lately emerged that some VNTR sequences may have significant roles in regulating transcription. For example, differences in monoamine oxidase A (MAOA) activity depend on the number of repeat units in a VNTR sequence located upstream of the MAOA gene (Sabol et al. 1998; Denney et al. 1999). Furthermore, Chevalier et al. (2001) have suggested that a four-allele VNTR sequence in the promoter region of the gene encoding cytochrome $\mathrm{P} 450$ prostacyclin synthase (CYP8A1) could account for individual differences in CYP8A1 transcription in vivo, in response to interleukin-6.

Some investigators have reported that alleles at certain VNTR loci are associated with the prevalence of diseases such as ovarian cancer (Phelan et al. 1996; Weitzel et al. 2000), progressive myoclonus epilepsy of UnverrichtLundborg type (Lafrenière et al. 1997; Alakurtti et al. 2000), and insulin-dependent diabetes mellitus (Lucassen et al. 1993; Pugliese et al. 1997; Vafiadis et al. 1997; Stead et al. 2000). A relationship may exist between the involvement of VNTRs in regulation of transcription and the association of some VNTR alleles with disease susceptibility.

In earlier work we found that cosmid clone cCI11p15-10 contained a VNTR sequence (Takita et al. 1992). Because genomic sequence analysis revealed that this VNTR sequence was located upstream of a gene corresponding to an expressed sequence tag, DKFZp434A0527, we chose to examine whether polymorphism of the repetitive sequence might influence the transcriptional activity. We show here that this VNTR sequence is polymorphic in the Japanese population, and that it can affect transcriptional activity of a promoter in vitro. Our data suggest that in vivo this VNTR sequence may influence transcription of DKFZp434A0527 in a manner dependent on the copy number of repetitive units present, causing differences among individuals as regards expression of this downstream gene, whose function is not yet known. 


\section{Materials and methods}

\section{Genotyping}

We synthesized two primers, V1 (5'-CCACCTGCCTACA TGGTATCTGC CTGAA-3') and V3 (5'-GACAGCACT CAGAGCAGCCTAGAGAC-3'), on the basis of the DNA sequence from cosmid clone cCI11p15-10, to amplify genomic DNA containing the VNTR. We carried out polymerase chain reactions (PCRs) using genomic DNA from 80 unrelated Japanese individuals. Each PCR was performed in a volume of $25 \mu \mathrm{l}$ containing $30 \mathrm{ng}$ genomic DNA, $8.7 \mathrm{mM} \mathrm{MgCl}_{2}$, PCR buffer $(67 \mathrm{mM}$ Tris at $\mathrm{pH} 8.8,16.6 \mathrm{mM}$ $\left(\mathrm{NH}_{4}\right)_{2} \mathrm{SO}_{4}, 6.7 \mu \mathrm{M}$ ethylene-diamine tetraacetic acid, $10 \mathrm{mM} \beta$-mercaptoethanol), $10 \mathrm{pmol}$ of each primer, $2.5 \mathrm{mM}$ of each deoxyribonucleotide triphosphate, $10 \%$ dimethylsulfoxide, and 1.0 unit of Ex Taq DNA polymerase (TaKaRa Shuzo, Otsu, Japan), in a Gene Amp PCR9700 (Applied Biosystems, Foster City, CA, USA). The cycle conditions were $96^{\circ} \mathrm{C}$ for $4 \mathrm{~min}$, followed by 35 cycles of $96^{\circ} \mathrm{C}$ for $30 \mathrm{~s}, 63^{\circ} \mathrm{C}$ for $30 \mathrm{~s}$, and $72^{\circ} \mathrm{C}$ for $4 \mathrm{~min}$. PCR products were separated by electrophoresis on $0.8 \%$ agarose gels, which were then stained with ethidium bromide.

\section{Construction of reporter gene for luciferase assay}

The four different VNTR alleles were cloned separately into the pGL3-Promoter vector (Promega, Madison, WI, USA), which had an SV40 promoter in front of a firefly luciferase gene. The sequence of each reporter construct was verified by the ABI 3700 autosequencer (Applied Biosystems).

\section{Cell culture and transfection assay}

A human testis-carcinoma cell line, NEC8 (obtained from RIKEN gene bank), was grown in RPMI1640 medium supplemented with $10 \%$ fetal bovine serum at $37^{\circ} \mathrm{C}$ in $5 \%$ $\mathrm{CO}_{2}$. The cells were plated on 60 -mm dishes $\left(2.0 \times 10^{5}\right.$ cells/ 60-mm dish) and transfected $48 \mathrm{~h}$ later. pRL-TK vector (Promega), in which Renilla luciferase coding sequence was fused to the Herpes simplex virus thymidine kinase promoter region, was cotransfected as an internal control. We mixed each reporter vector with the internal control vector, and added the mixtures to $100 \mu$ l OPTI-MEM I medium (GIBCO BRL, Rockville, MD, USA) containing $6 \mu \mathrm{l}$ FuGENE6 transfection reagent (Boehringer Mannheim, Mannheim, Germany). This medium was incubated for $15 \mathrm{~min}$ and then added to the NEC8 cultures. After $48 \mathrm{~h}$ of further incubation, the transfected cells were washed with phosphate-buffered saline and lysed with $500 \mu l$ of passive lysis buffer (Promega). Lysate proteins were retrieved in tubes and centrifuged briefly to remove cellular debris. Ten microliters of each extract was sequentially assayed for firefly and Renilla luciferase activities using the DualLuciferase Reporter System (Promega) according to the manufacturer's instructions. Luminescence measurements were carried out with a Lumat LB9507 luminometer (Berthold Technologies, Bad Wildbad, Germany). Results were expressed as the ratio of activity of firefly to Renilla luciferase. All transfections were carried out in duplicate or triplicate, and each construct was tested in at least five independent experiments using separate preparations of plasmid DNA.

\section{Statistical analysis}

Transfection results were expressed as mean \pm SD. Analyses of variance (ANOVA) and subsequent Scheffé's tests were used to determine significance of the differences in multiple comparisons. A difference in promoter activity among reporter constructs was considered significant statistically when the $P$ value was less than 0.05 .

\section{Results}

\section{VNTR upstream of cDNA DKFZp434A0527}

We previously reported that cosmid clone cCI11p $15-10$ contained a VNTR sequence (Takita et al. 1992). Sequence analysis of this cosmid clone disclosed a 38-bp consensus sequence of the repeating unit (CCTTGGTGAGGGGAC CTGGTGAGGGGACATGGGGGGGA) with some variations in length (38-41 bp) and DNA sequence from one unit to another (Fig. 1). A search for homology of this VNTR sequence with published genomic sequences revealed that it was located 125 nucleotides upstream of sequences corresponding to the $5^{\prime}$ end of a human cDNA, DKFZp434A0527 (Fig. 1).

To investigate polymorphism of this VNTR sequence, we synthesized a pair of PCR primers (V1 and V3, Fig. 1) corresponding to DNA sequences flanking the VNTR sequence, and performed PCR experiments using genomic DNA preparations from 80 unrelated individuals as templates. We detected four different alleles in this population sample: $750 \mathrm{bp}, 900 \mathrm{bp}, 1.2 \mathrm{~kb}$, and $1.5 \mathrm{~kb}$ (designated as alleles A1, A2, A3, and A4, respectively). Estimated allelic frequencies are listed on Table 1; 11 of the 80 individuals were heterozygous at this locus. Sequence analysis of the 750 bp fragment (allele A1) indicated that this allele contained ten copies of the repetitive unit, but we were unable to determine the precise number of repetitive units in the other three alleles because DNA sequencing was unsuccess-

Table 1. Frequency and size of VNTR alleles at human chromosome $11 \mathrm{p} 15$

\begin{tabular}{lllr}
\hline & & $\begin{array}{l}\text { Allele frequency } \\
(n=160)\end{array}$ & Number of alleles \\
\hline Allele & Allele size $(\mathrm{kb})$ & 0.794 & 127 \\
A2 & 0.75 & 0.144 & 23 \\
A3 & 0.9 & 0.031 & 5 \\
A4 & 1.2 & 0.031 & 5 \\
Total & - & 1.000 & 160
\end{tabular}

VNTR, variable number of tandem repeat 
v1 primer

-641 CCACCTGCCTACATGGTATCTGCCTGAATTTCCATTTGGCTGAGGGAGCCCTAGGGAGGGGGCC

-577 CTGGAGGACTTGGTTACAGGGGACCTGGTGAGGGGACCCAGTGAGGGGGACTTCATGGGTGGGTG

$\begin{array}{lll}-512 & \text { CCTGGTTGAGGGGACCTGGTGAAAGAACATGGTGAGGGGG } & \text { repeat } \mathbf{1} \\ -471 & \text { CCTGGTGAGGGGTGGTCTGGTGAGGGAACTTAGGGAAA } & \text { repeat } \mathbf{2} \\ -433 & \text { CCTGGTGAGGAGGACCTGGTGAGGGGACATGGGGGGGA } & \text { repeat } \mathbf{3} \\ -395 & \text { CCTTGGTGAGGGGACCTGGTGAGGGGACATAGGGGGA } & \text { repeat } \mathbf{4} \\ -358 & \text { CCTTGGTGAGCGGACCTGGTGAGGGGACATGGGGGGGA } & \text { repeat } \mathbf{5} \\ -320 & \text { CCTTGGTGAGGGGACCTGGTGAGGAGGACATGGGGGGGA } & \text { repeat } \mathbf{6} \\ -281 & \text { CCTTGGTGAGGGGACCTGGTGAGGGGACATGGGGGGGA } & \text { repeat } \mathbf{7} \\ -243 & \text { CCTTGGTGAGGGGACCTGGTGAGGAGGACATGGGGGGGA } & \text { repeat } \mathbf{8} \\ -204 & \text { CCTTGGTGAGGGGACCTGTAGGGGGACCTATGGGGGGGGA } & \text { repeat } \mathbf{9} \\ -164 & \text { CCTTGTTGGGGGGACCTGGTGAGGAGATCTGGTGAGGGGA } & \text { repeat } \mathbf{1 0}\end{array}$

-124 TCAAAGGCAGAAGCCATTGTCTTGGTCACATAGACAGAATCTAGAACCCGTCATCCCACAGAGCCACATTCTAGAGTTTAGAGTGGGGGA
+1 CDNA DKFZM434A0527

-34 CCTCTCCCCAATTCTGTAAGCCCATGAGGAGAGAGGAAGGCCTTTCTCTCCATGCTCCTTTTGCGGGAACTCCCTGGCACCAAGGCCAGG

+57 GGGGAAAGGACAGTCTCTGATGAGGATGGGTCTCTAGGCTGCTCTGAGTGCTGTC

v3 primer

\section{Consensus sequence 5'-CCTTGGTGAGGGGACTGGTGAGGGGACATGGGGGGGA-3'}

Fig. 1. Nucleotide sequence corresponding to allele A1 of the variable number of tandem repeat (VNTR) sequence, and the consensus sequence of the repetitive unit. Arrows indicate the locations of primers used to amplify the minisatellite. Numbers at the left indicate positions relative to the 5' end of human cDNA DKFZp434A0527 ful, probably because of a very high proportion of $\mathrm{G} / \mathrm{C}$ nucleotides.

In vitro analysis of the effect of the VNTR sequence on promoter activity

This VNTR sequence appeared to be located in the promoter region of DKFZp434A0527, although the function of this gene is unknown. Considering that this VNTR sequence was likely to be located in the promoter region (although the possibility of being located in an intron was not excluded) and that some VNTRs located near promoter regions or in introns are known to play significant roles in transcriptional regulation, we suspected that this VNTR sequence might have a similar influence on promoter activity. To verify this hypothesis, we cloned each of the four alleles of this VNTR sequence into a luciferase vector (pGL3-Promoter vector) and examined the effect that different numbers of repeat units would have on transcrip- tional activity in the reporter assay after transient transfection into a testis-carcinoma cell line, NEC8.

All constructs that contained one of the VNTR alleles suppressed the transcriptional activity of the SV40 promoter present in the pGL3-Promoter vector. We also made a construct containing a $750 \mathrm{bp}$ genomic DNA fragment without any repetitive sequences and performed the same experiment as a control, but observed no effect on the transcriptional activity of the reporter gene (Fig. 2). There were distinct differences in transcriptional activity between this control construct and each VNTR allele $(P<0.0001)$. The greater the number of repetitive units present, the more the transcriptional activity was suppressed; i.e., the rare, longer alleles (A3 or A4) seemed to suppress the promoter activity of the reporter gene more than did the major, shorter alleles (A1 and A2) (A4 vs. A1 and A2; $P<0.0001$; A3 vs. A1; $P=0.0002 ; \mathrm{A} 3$ vs. A2; $P=0.0065)$. On the other hand, the difference in promoter activities between alleles A1 and A2 was not statistically significant $(P=0.8340)$, nor was the difference between alleles A3 and A4 $(P=0.3866)$. 
Fig. 2. Influence of the VNTR sequence on promoter activity in transiently transfected NEC8 cells. The diagram indicates the luciferase activity of each VNTR allele, corrected for transfection efficiency and recorded relative to that of the pGL3-Promoter vector alone. Mean $\pm \mathrm{SD}$ values for at least five independent experiments are shown. $P$ indicates SV40 promoter that is present in the pGL3-Promoter vector. The hatched box denotes a 750-bp genomic DNA fragment containing no repetitive sequences. ${ }^{*} P<$ 0.0001 , alleles $\mathrm{A} 1$ and $\mathrm{A} 2$ vs. pGL3-Promoter vector or control; $* * P<0.0001$, alleles A3 and A4 vs. pGL3-Promoter vector or control, and $P<0.05$ for alleles A3 and A4 vs. alleles A1 and A2

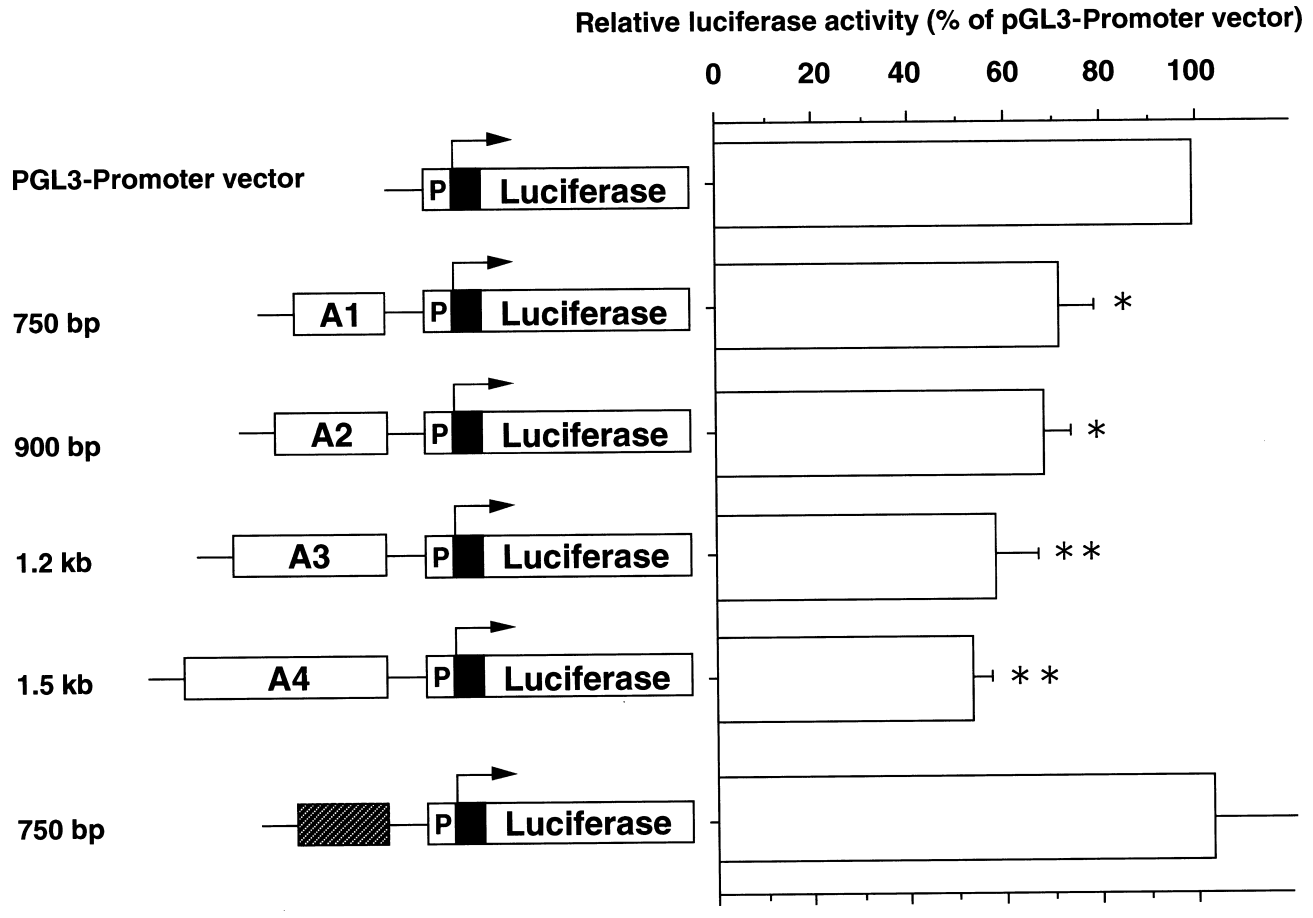

\section{Discussion}

We reported previously that six cosmid clones on human chromosome 11p15 contained VNTR sequences (Takita et al. 1992). DNA sequencing of these VNTRs and comparisons with genomic sequences disclosed that one VNTR sequence was likely to be located in the promoter region of a gene of unknown function, designated DKFZp434A0527. Reverse transcriptase-PCR experiments using this human cDNA as a template revealed that the gene was expressed in human testis and kidney (data not shown).

In a Japanese population sample of 160 chromosomes, we detected four different alleles of this VNTR sequence. The most common allele (A1) contained ten copies of the repetitive unit. Although we were unable to obtain the complete DNA sequences of the other three alleles for technical reasons, the copy numbers of the repetitive units were speculated to be 14 (A2), 22 (A3), and 30 (A4) on the basis of fragment lengths estimated by gel electrophoresis.

In vitro analysis of the effect of the different alleles on transcriptional activity was carried out using six plasmid clones, four of which contained a single allele of the VNTR sequence. Each clone was transfected into NEC8 cells and luciferase activity was measured. Alleles A3 and A4, corresponding to larger numbers of repeat units, suppressed transcriptional activity more significantly than did the shorter alleles (A1 and A2). When we compared the $750 \mathrm{bp}$ DNA fragment containing this VNTR sequence with 750 bp of genomic DNA containing no repeat sequence, only the fragment containing the VNTR sequence was able to suppress transcription. Because we obtained similar results in experiments using HeLa cells (data not shown), we suspected that this VNTR sequence was likely to func- tion as a transcriptional regulator in a nontissue specific manner.

The mechanism by which the major alleles (A1 and A2) and minor alleles (A3 and A4) influence transcriptional activity to different extents is unknown. Some transcription factors are known to bind to VNTR sequences. For example, members of the rel/NF-kB family of transcription factors bind VNTR sequences present in the $3^{\prime}$ flanking region of the HRAS gene; the consensus sequence of the repeat units in that VNTR sequence is highly homologous to the binding site of rel/NF-kB proteins (Trepicchio and Krontiris 1992). The same authors (1993) reported that although the VNTR sequence present in the human immunoglobulin heavy chain gene $(I G H)$, which contains a sequence similar to the motif for binding the myc/HLH transcription factor, had no enhancer activity, it was able to significantly suppress transcriptional activity of the adenovirus major late promoter. We speculate that some transcription factor(s) might bind to the repetitive sequence reported here, although at present we have no data to support this idea.

Associations of certain VNTRs with the risk of diseases have also been documented (reviewed by Nakamura et al. 1998). The presence of one or two of the rare alleles of the $H R A S$ VNTR sequence in a single individual appear to be associated with a higher risk of cancers in multiple tissues, e.g., colon, breast, and urinary bladder (Krontiris et al. 1993). Phelan et al. (1996) suggested that the status of the $H R A S$ VNTR sequence might influence the penetrance of ovarian cancer in individuals who carried a mutation in the breast-cancer susceptibility gene BRCA1. Considering that the novel VNTR sequence reported here seems to be located in the promoter region of a cDNA (DKFZp434A0527) that is expressed in testis and kidney, 
and is able to regulate in vitro transcriptional activity in a manner that depends on the number of repeats present, one or two of the rare VNTR alleles in a single individual might reduce expression of this gene and influence the individual's susceptibility to diseases involving testis or kidney.

In conclusion, our study has demonstrated polymorphism of this VNTR sequence among Japanese individuals and shown that its alleles could be ranked in two classes according to allele frequency and the effect on transcriptional activity. We have not determined the mechanism responsible for differences in transcriptional effect pertaining to each allele, but it is possible that this VNTR sequence might contain the binding site of a transcriptional regulator and the numbers of such units could thereby influence the efficiency of transcription.

Acknowledgments This work was supported in part by Research for the Future Program Grant \#00L01402 from the Japan Society for the Promotion of Science.

\section{References}

Alakurtti K, Virtaneva K, Joensuu T, Palvimo JJ, Lehesjoki AE (2000) Characterization of the cystatin $\mathrm{B}$ gene promoter harboring the dodecamer repeat expanded in progressive myoclonus epilepsy, EPM1. Gene 242:65-73

Chevalier D, Cauffiez C, Bernard C, Lo-guidice JM, Allorge D, Fazio F, Ferrari N, Libersa C, Lhermitte M, D'Halluin JC, Broly F (2001) Characterization of new mutations in the coding sequence and 5 -untranslated region of the human prostacycin synthase gene (CYP8A1). Hum Genet 108:148-155

Denney RM, Koch H, Craig IW (1999) Association between monoamine oxidase A activity in human male skin fibroblasts and genotype of the MAOA promoter-associated variable number tandem repeat. Hum Genet 105:542-551

Krontiris TG, Devlin B, Karp DD, Robert NJ, Risch N (1993) An association between the risk of cancer and mutations in the HRAS1 minisatellite locus. N Engl J Med 329:517-523

Lafrenière RG, Rochefort DL, Chrétien N, Rommens JM, Cochius JI, Kälviäinen R, Nousiainen U, Patry G, Farrell K, Söderfeldt B,
Federico A, Hale BR, Cossio OH, Sørensen T, Pouliot MA, Kmiec T, Uldall P, Janszky J, Pranzatelli MR, Andermann F, Andermann E, Rouleau GA (1997) Unstable insertion in the 5' flanking region of the cystatin B gene is the most common mutation in progressive myoclonus epilepsy type 1, EPM1. Nat Genet 15:298-302

Lucassen AM, Julier C, Beressi JP, Boitard C, Froguel P, Lathrop M, Bell JI (1993) Susceptibility to insulin dependent diabetes mellitus maps to a $4.1 \mathrm{~kb}$ segment of DNA spanning the insulin gene and associated VNTR. Nat Genet 4:305-310

Nakamura Y, Leppert M, O'Connell P, Wolff R, Holm T, Culver M, Martin C, Fujimoto E, Hoff M, Kumlin E, White R (1987) Variable number of tandem repeat (VNTR) markers for human gene mapping. Science 235:1616-1622

Nakamura Y, Koyama K, Matsushima M (1998) VNTR (variable number of tandem repeat) sequences as transcriptional, translational, or functional regulators. J Hum Genet 43:149-152

Phelan CM, Rebbeck TR, Weber BL, Devilee P, Ruttledge MH, Lynch HT, Lenoir GM, Stratton MR, Easton DF, Ponder BAJ, CannonAlbright L, Larsson C, Goldgar DE, Narod SA (1996) Ovarian cancer risk in BRCA1 carriers is modified by the HRAS1 variable number of tandem repeat (VNTR) locus. Nat Genet 12:309-311

Pugliese A, Zeller M, Fernandez A Jr, Zalcberg LJ, Bartlett RJ, Ricordi C, Pietropaolo M, Eisenbarth GS, Bennett ST, Patel DD (1997) The insulin gene is transcribed in the human thymus and transcription levels correlate with allelic variation at the INS VNTRIDDM2 susceptibility locus for type 1 diabetes. Nat Genet 15:293297

Sabol SZ, Hu S, Hamer D (1998) A functional polymorphism in the monoamine oxidase A gene promoter. Hum Genet 103:273-279

Stead JDH, Buard J, Todd JA, Jeffreys AJ (2000) Influence of allele lineage on the role of the insulin minisatellite in susceptibility to type 1 diabetes. Hum Mol Genet 9:2929-2935

Takita K, Tanigami A, Tokino T, Jones C, Nakamura Y (1992) Identification of 57 conventional RFLP and 6 VNTR systems with 32 DNA clones on chromosome 11p15. Genomics 13:1296-1299

Trepicchio WL, Krontiris TG (1992) Members of the rel/NFkB family of transcriptional regulatory proteins bind the $H R A S 1$ minisatellite DNA sequence. Nucleic Acids Res 20:2427-2434

Trepicchio WL, Krontiris TG (1993) IGH minisatellite suppression of USF-binding-site- and E $\mu$-mediated transcriptional activation of the adenovirus major late promoter. Nucleic Acids Res 21:977-985

Vafiadis P, Bennett ST, Todd JA, Nadeau J, Grabs R, Goodyer CG, Wickramasinghe S, Colle E, Polychronakos C (1997) Insulin expression in human thymus is modulated by INS VNTR alleles at the IDDM2 locus. Nat Genet 15:289-292

Weitzel JN, Ding S, Larson GP, Nelson RA, Goodman A, Grendys EC, Ball HG, Krontiris TG (2000) The HRAS1 minisatellite locus and risk of ovarian cancer. Cancer Res 60:259-261 